\title{
Epithelial-mesenchymal transition (EMT) in vulvar cancer with and without inguinal lymph node involvement
}

\author{
Christine E. Brambs ${ }^{1}$. Lars-Christian Horn ${ }^{2}$ - Meinhard Mende ${ }^{3} \cdot$ Michael Höckel $^{4}$. Christine Eckey ${ }^{2}$. \\ Gesine Grit Ruth Hiller ${ }^{2}$ Anne Kathrin Höhn²
}

Received: 4 March 2021 / Accepted: 24 June 2021 / Published online: 8 September 2021

(c) The Author(s) 2021

\begin{abstract}
Purpose Epithelial-mesenchymal transition (EMT) is associated with increased metastatic spread and poor prognosis. Data on vulvar carcinoma are limited.

Methods Thirty-two cases of squamous cell carcinoma of the vulva (16 with and 16 without inguinal lymph node metastases) and their lymph node deposits were evaluated for immunohistochemical expression of EMT markers (vimentin, cyclin D1, e-cadherin), p16, p53 and Ki-67. Results of EMT-immunostainings were compared to lymph node involvement and expression of p53 and p16. The micro-anatomical staining pattern for EMT markers comparing the tumor center with the front of invasion was analysed in each tumor.

Results There was no difference in the expression of EMT markers between node negative and node positive tumors. Staining for vimentin and cyclin D1 was seen within tumor cells at the front of invasion in 100 and $84.4 \%$ of the tumors, respectively. The majority of cases $(68.7 \%$ ) showed negative or reduced staining for e-cadherin in this micro-anatomical localization. Tumor cells within the lymph node metastases showed positive staining for e-cadherin in $75 \%$ and for cyclin D1 in $49 \%$ of the cells but were negative for vimentin in 13 out of 16 cases (81.3\%). Tumors with aberrant p53 staining represented a non-significant higher vimentin but significantly higher cyclin D1 expression at the front of invasion than those with p53 wild-type pattern.

Conclusion The present study shows no differences in the expression of EMT markers between node positive and node negative vulvar cancers. The evaluation of immunostaining within the micro-anatomical context indicates that an EMT-phenotype is restricted to the tumor cells at the front of invasion. Paired analyses of vulvar carcinomas and their lymph node deposits suggest mesenchymal-epithelial transition (MET) in the metastatic deposits. Immunohistochemical staining results may suggest that EMT is more prevalent in vulvar cancer with aberrant p53 staining.
\end{abstract}

Keywords Vulvar cancer $\cdot$ EMT $\cdot$ p53 $\cdot$ p16 Epithelial $\cdot$ Mesenchymal transition · Front of invasion · Cyclin D1 . Vimentin $\cdot$ e-cadherin $\cdot$ Mesenchymal-epithelial transition $\cdot$ MET

\section{Introduction}

Vulvar cancer is a rare gynaecologic malignancy accounting for 2-5\% of all gynaecologic cancers (Rogers and Cuello

ChristineE. Brambs and Lars-Christian Horn both authors contributed equally to the paper

Lars-Christian Horn

hornl@medizin.uni-leipzig.de

1 Department of Obstetrics and Gynecology, Luzerner Kantonsspital, Lucerne, Switzerland

2 Institute of Pathology, Division of Breast Gynecologic and Perinatal Pathology, University Hospital of Leipzig, Liebigstrasse 26, 04103 Leipzig, Germany
2018; Te Grootenhuis et al. 2018). Although it mostly affects elderly women, the incidence in younger patients is rising (Te Grootenhuis et al. 2018).

Vulvar squamous cell carcinoma (VSCC) is the most common histopathological subtype of vulvar cancer (Dasgupta et al. 2020; Rogers and Cuello 2018; Singh and Gilks

Clinical Trail Centre and Institute of Medical Information, Statistics and Epidemiology, University of Leipzig, Leipzig, Germany

4 Division of Gynecologic Surgical Oncology, Department of Obstetrics and Gynecology (Institute of Trier), University Hospital of Leipzig, Leipzig, Germany 
2020). There are two distinct molecular pathways that lead to VSCC, associated with either (1) VIN of the usual type (u-VIN) which is associated with high-risk HPV infections, mostly resulting in non-keratinizing VSCC and (2) VIN of the differentiated type (d-VIN), associated with p53 alterations often developing in a background of lichen sclerosus, mostly resulting in keratinizing VSCC (Dasgupta et al. 2020; Singh and Gilks 2020).

Regardless of the pathogenetic pathway, one of the most relevant prognostic factors for local recurrence of VSCC is the presence of inguinal lymph node metastases (Rogers and Cuello 2018; Te Grootenhuis et al. 2018). Risk factors for lymph node involvement of VSCC are the depth of invasion, tumor size and the ontogenetic tumor stage (Höckel et al. 2018; Julia and Hoang 2021) as well as morphologic features, such as infiltrative growth with dissociatively growing tumors and the presence of strong peritumoral desmoplastic change (Holthoff et al. 2016; Horn et al. 2012). Dissociative tumor growth and strong peritumoral stromal remodelling have been shown to be associated with epithelial-to-mesenchymal transition (EMT) in VSCC (Holthoff et al. 2016; Rodrigues et al. 2013). Furthermore, there is mounting evidence that EMT plays an essential role in lymphatic spread (Campo et al. 2015).

The present study was designed to evaluate the immunohistochemical expression of EMT markers in correlation to the presence of inguinal lymph node involvement in VSCC.

\section{Materials and methods}

Patients diagnosed with macro-invasive squamous carcinomas of the vulva were identified from our medical records. All patients had no previous vulvar surgery, had not received neoadjuvant therapy and were included in the prospective surgical trial for vulvar field resection based on the ontogenetic anatomy (German Clinical Trials Register, number DRKS00013358; (Höckel et al. 2018)). Patients were separated into those without and those with histopathologically proven inguinal lymph node involvement. Representative tumor tissue, using full slides, was evaluated immunohistochemically for p53, p16, MIB-1 and EMT markers (vimentin, cyclin D1, e-cadherin). Immuno-histochemical antibody information is provided in Table 1.

p16 was scored as positive if there was diffuse block-like cytoplasmic and nuclear staining and as negative for any lesser staining (such as patchy or absent staining) in accordance with the LAST (Lower Anogenital Squamous Terminology) project recommendations (Darragh et al. 2013) and recent studies (Tessier-Cloutier et al. 2020).

p53 was scored as wild type representing heterogeneous nuclear staining of variable intensity of scattered nuclearpositive tumor cells in the basal and parabasal layers and
Table 1 Immuno-histochemical antibody information

\begin{tabular}{|c|c|c|c|c|}
\hline Antibody & Clone & Vendor & $\begin{array}{l}\text { Dilution and } \\
\text { pretreatment }\end{array}$ & $\begin{array}{l}\text { Detection } \\
\text { system }\end{array}$ \\
\hline p53 & Do-7 & Dako & $\begin{array}{l}1: 100 \\
\text { CC1 36'/32, }\end{array}$ & DAB \\
\hline p16 & E6H4 & $\begin{array}{l}\text { Cintec Histol- } \\
\text { ogy }\end{array}$ & $\begin{array}{l}\text { ready to use } \\
\text { CC1 } 36^{\prime} / 32\end{array}$ & $\mathrm{DAB}$ \\
\hline cyclin D1 & Sp4 & Zytomed & $\begin{array}{l}1: 50 \\
\text { CC1 } 64 \text { ‘/32، }\end{array}$ & DAB \\
\hline vimentin & Vim3B4 & Dako & $\begin{array}{l}1: 200 \\
\text { CC1 } 36 \text { ‘/32، }\end{array}$ & Ultraview \\
\hline e-cadherin & $36 \mathrm{~b} 5$ & Novocastra & $\begin{array}{l}1: 20 \\
\text { CC1 } 76 \text { ‘/32، }\end{array}$ & Ultraview \\
\hline MIB-1 & MIB-1 & DAKO & $\begin{array}{l}1: 100 \\
\text { CC1 } 64 \text { ‘/32، }\end{array}$ & DAB \\
\hline
\end{tabular}

mid-epithelial with heterogeneously positively stained nuclei with notable basal negativity of the invasive tumor cell nests. Mutant staining patterns were defined as strong nuclear staining in at least $80 \%$ of the basal cells with or without significant parabasal extension, complete negative staining of the tumor cells with the presence of a positive staining in adjacent inflammatory and stromal cells (serving as positive internal control) as described recently for VSCC (Tessier-Cloutier et al. 2020).

Nuclear staining of Ki-67 was scored from 1-3 (Fig. 1) on the basis of a comparison with normal vulvar squamous epithelium (Podoll et al. 2017) and existing evidence on penile squamous cell carcinoma (Protzel et al. 2007; Stankiewicz et al. 2012): 1 = basal expression or low-grade pattern with nuclear Ki-67 staining of the basal/ outer cell layers of the invasive tumor cells nests with very limited suprabasal extension; 2 =increased expression or intermediate pattern with staining up to the middle of the infiltrating tumor cell nests; $3=$ increased expression or high-grade pattern with diffuse staining of the tumor cell nests. All nuclear staining was recognised as positive, regardless of the staining intensity.

For EMT markers, an immuno-reactive score (IRS) was calculated by summarizing the percentage of positive cells and their staining intensity. Staining intensity (SI) was scored as weak, moderate or strong. The percentage of positive tumor cells at the front of invasion was scored as $0=0 \%$ positive tumor cells, $1=1 \%$ positive cells, $2=2-<10 \%$ positive cells, $3=10-<33 \%$ positive cells, $4=33-<66 \%$ positive cells, $5=66-100 \%$ positive cells. The median values of IRS were evaluated in correlation to the presence of inguinal lymph node involvement. Additionally, the zonal staining pattern was evaluated, comparing the front of invasion with the tumor center (Stewart and McCluggage 2013).

The pattern of invasion and the grade of peritumoral desmoplastic reaction (DSR) and inflammatory response (PER) 


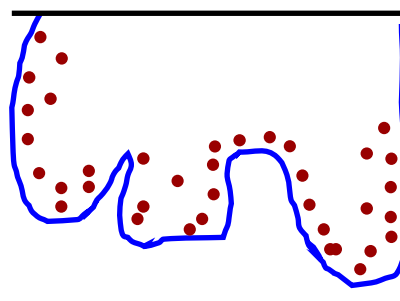

low-grade pattern (score 1)

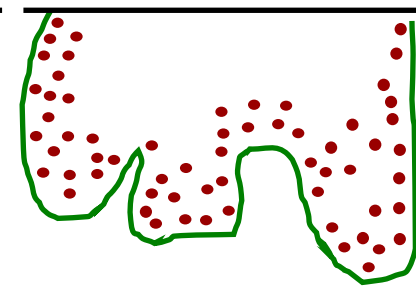

intermediate pattern (score 2)

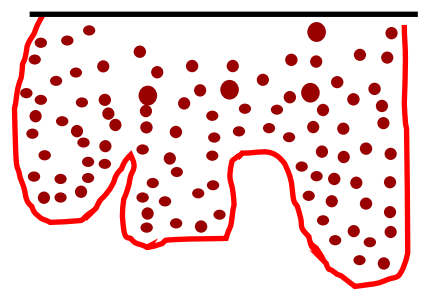

high-grade pattern (score 3)
Fig. 1 Schematic illustration of Ki-67 scoring system: Score $1=$ lowgrade pattern: positive staining of the outer cell layers of the invasive tumor cells nests with very limited suprabasal extension. Score

were evaluated in correlation to the lymph node status as previously described (Horn et al. 2012).

The study cohort was described by standard descriptive statistics: median for continuous, number for categorical variables. Relationships between categorical variables were analysed by crosstabs, chi-squared tests and Fisher's exact tests as appropriate. The location was compared by nonparametric tests due to the low sample size and asymmetrical distribution of the scores, that is, Mann-Whitney $U$ test for two and Kruskal-Wallis test for more than two groups.

Median differences including 95\% confidence intervals were calculated as effect measures following Hodges and Lehman. The significance level was determined at $5 \%$ for two-tailed tests. All analyses were performed by IBM SPSS Statistics, version 26.

An Institutional Review Board approval was obtained prior to the initiation of the study.

\section{Results}

\section{Clinicopathological data}

Thirty-two cases were included in the analysis. Their clinico-pathological characteristics are summarised in Table 2.

Patients with inguinal lymph node involvement were in median six years older compared to patients without metastatic spread (68.6 vs. 74.3 years; Table 2).

About one half of the tumors showed strong peritumoral desmoplastic change (DES; Fig. 2a). There were no differences between the grade of DES when node positive and negative cases were compared $(p=1.0)$.

$56.3 \%$ of the node positive tumors showed no or an only weak peritumoral inflammatory response (PER; Fig. 2b), whereas $68.8 \%$ of the carcinomas without inguinal lymph node involvement ( $\mathrm{pN} 0$ ) represented with a moderate or strong PER (Fig. 2c) without reaching statistical
$2=$ intermediate pattern: positive staining up to the mid of the infiltrating tumor cell nests. Score $3=$ high-grade pattern: with diffuse or near-diffuse staining of the tumor cell nests

significance $(p=0.26)$. The different patterns of invasion were not associated with the lymphatic spread (Table 2).

\section{Proliferate activity and p53- and p16-immunostaining}

More than $60 \%$ of the VSCC without inguinal lymph node involvement showed a low proliferative activity (Ki-67 score 1), whereas one-third of the tumors with positive inguinal nodes were highly proliferative, with a Ki-67 score of 3 (Table 2, Fig. 3).

About two-thirds of tested VSCC represented aberrant p53 staining which is suggestive of a p53 mutation (Tessier-Cloutier et al. 2020; Table 3, Fig. 4a). One-third showed p16 block staining which has been reported to be associated with high-risk HPV infections (Tessier-Cloutier et al. 2020; Table 3, Fig. 4b). One single case showed both, aberrant p53 expression accompanied by block-p16-positivity (Fig. 4c), representing a so-called double classifier (Tessier-Cloutier et al. 2020). That special case was included in further analysis of the EMT-marker expression as p16-positive one.

Although a limited number of cases were included in this study, those with an immuno-histochemical signature of $\mathrm{p} 16^{\mathrm{block}} / \mathrm{p} 53^{\mathrm{wt}}$, indicating an HPV association, represented a significantly higher number of highly proliferative tumors characterized by a Ki-67 score of 3 (Fig. 5a). In contrast, the

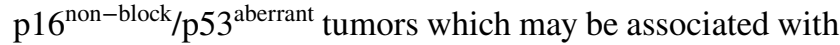
a p53 mutation, showed a low proliferative activity based on a Ki-67 score of 1 (Fig. 5a; Table 3). There were significantly more spray-like patterns of invasion in the p53-aberrant compared to the p16-block-positive tumors (Fig. 5b).

\section{Immunostaining of EMT markers}

No differences within the median values of the immunoreactive scores (IRS) could be proven when comparing node 
Table 2 Patient characteristics

\begin{tabular}{|c|c|c|c|c|}
\hline & \multicolumn{4}{|c|}{ Inguinal lymph node status } \\
\hline & All cases $(\mathrm{N}=32)$ & $\mathrm{pN} 0(N=16)$ & $\mathrm{pN} 1(N=16)$ & \\
\hline Median age (years) & $70.7(39.8-87.5)$ & $68.6(39.8-77.7)$ & $74.3(40.5-87.5)$ & $p=0.017$ \\
\hline \multicolumn{5}{|l|}{ Tumor stage } \\
\hline pT1b & $16(50.0 \%)$ & $8(50.0 \%)$ & $8(50.0 \%)$ & \\
\hline pT2 & $10(31.2 \%)$ & $4(25.0 \%)$ & $6(37.5 \%)$ & \\
\hline pT3 & $6(18.8 \%)$ & $4(25.0 \%)$ & $2(12.5 \%)$ & $p=0.59$ \\
\hline \multicolumn{5}{|l|}{ Tumor grade } \\
\hline G1 & $2(6.3 \%)$ & $2(12.5 \%)$ & 0 & \\
\hline G2 & $20(62.5 \%)$ & $9(56.3 \%)$ & $11(68.8 \%)$ & \\
\hline G3 & $10(31.2 \%)$ & $5(31.2 \%)$ & $5(31.2 \%)$ & $\mathrm{p}=0.61$ \\
\hline \multicolumn{5}{|c|}{ Peritumoral desmoplastic change } \\
\hline None & $4(12.5 \%)$ & $2(12.5 \%)$ & $2(12.5 \%)$ & \\
\hline Weak & $10(31.3 \%)$ & $5(31.3 \%)$ & $5(31.3 \%)$ & \\
\hline Moderate & $3(9.3 \%)$ & $1(6.2 \%)$ & $2(12.5 \%)$ & \\
\hline Strong & $15(46.9 \%)$ & $8(50.0 \%)$ & $7(43.7 \%)$ & $\mathrm{p}=1.0$ \\
\hline \multicolumn{5}{|c|}{ Peritumoral inflammatory response } \\
\hline None & $8(25.0 \%)$ & $4(25.0 \%)$ & $4(25.9 \%)$ & \\
\hline Weak & $6(18.8 \%)$ & $1(6.3 \%)$ & $5(31.3 \%)$ & \\
\hline Moderate & $10(31.2 \%)$ & $7(43.8 \%)$ & $3(18.7 \%)$ & \\
\hline Strong & $8(25.0 \%)$ & $4(25.0 \%)$ & $4(25.0 \%)$ & $p=0.26$ \\
\hline \multicolumn{5}{|l|}{ Pattern of invasion } \\
\hline Spray-like & $19(59.4 \%)$ & $10(62.5 \%)$ & $9(56.3 \%)$ & \\
\hline Finger-like & $13(40.6 \%)$ & $6(37.5 \%)$ & $7(43.8 \%)$ & $p=1.0$ \\
\hline \multicolumn{5}{|l|}{ p53-staining ${ }^{\mathrm{a}}$} \\
\hline Aberrant & $20(64.5 \%)^{\mathrm{b}}$ & $11(68.7 \%)$ & $9(60.0 \%)$ & \\
\hline Wild type & $11(35.5 \%)$ & $5(31.3 \%)$ & $6(40 \%)$ & $p=0.61$ \\
\hline \multicolumn{5}{|l|}{ p16-staining ${ }^{\mathrm{a}}$} \\
\hline Block & $12(38.7 \%)^{\mathrm{b}}$ & $5(31.2 \%)$ & $7(46.7 \%)$ & \\
\hline Non-block & $19(61.3 \%)$ & $11(68.7 \%)$ & $8(53.3 \%)$ & $p=0.38$ \\
\hline \multicolumn{5}{|l|}{ Ki-67-staining ${ }^{\mathrm{a}}$} \\
\hline Score 1 & $16(51.6 \%)$ & $10(66.7 \%)$ & $6(40.0 \%)$ & \\
\hline Score 2 & $7(22.6 \%)$ & $3(20.0 \%)$ & $4(26.7 \%)$ & \\
\hline Score 3 & $8(25.8 \%)$ & $3(13.3 \%)$ & $5(33.3 \%)$ & $p=0.52$ \\
\hline
\end{tabular}

${ }^{\mathrm{a}}$ In one case no tumor tissue was left within the only available tumor containing block for immunostaining

${ }^{\mathrm{b}}$ One single case represented aberrant p53 expression as well as block-p16-positivity within the tumor (Fig. 4c,d)

negative and positive cases for vimentin [pN0: 2.5 (95\% CI: $0.0-6.5$ ) versus $\mathrm{pN} 1: 3.5$ (95\% CI: $0.0-5.5) ; p=0.93$ ] and cyclin D1 [pN0: 5.5 (95\% CI: 3.0-7.5) versus pN1: 5.5 (95\% CI: $4.0-7.5) ; p=1.0]$ or for e-cadherin $(p=1.0)$. Characteristic staining results of the different EMT markers within the VSCC are illustrated in Figs. 6a-c.

Although not reaching statistical significance, VSCC with aberrant p53 staining showed a higher vimentin expression within the tumor cells $(p=0.087)$. In contrast, $\mathrm{p} 53$-aberrant tumors showed an increased cyclin D1 expression compared to p16-positive tumors ( $p=0.027$; suppl. Table 1$)$, while no difference was noted for e-cadherin $(p=1.0)$.
Evaluating the EMT markers within the lymph node metastases, positive staining was seen in all nodal tumor deposits for e-cadherin with an average of $74.9 \%$ positive tumor cells. While one case was completely negative for cyclin D1, positive staining was observed an average of $48.7 \%$ of positive stained tumor cells in all other cases (Fig. 6d-f). Only three metastatic deposits within the inguinal lymph nodes showed positive staining for vimentin. One of these cases presented with a very small metastasis of $0.25 \mathrm{~mm}$ in largest dimension (Fig. $6 \mathrm{~g}, \mathrm{~h}$ ). That staining pattern may suggest mesenchymal-epithelial transition (MET) within the metastatic tumor cells in the lymph node deposits. 


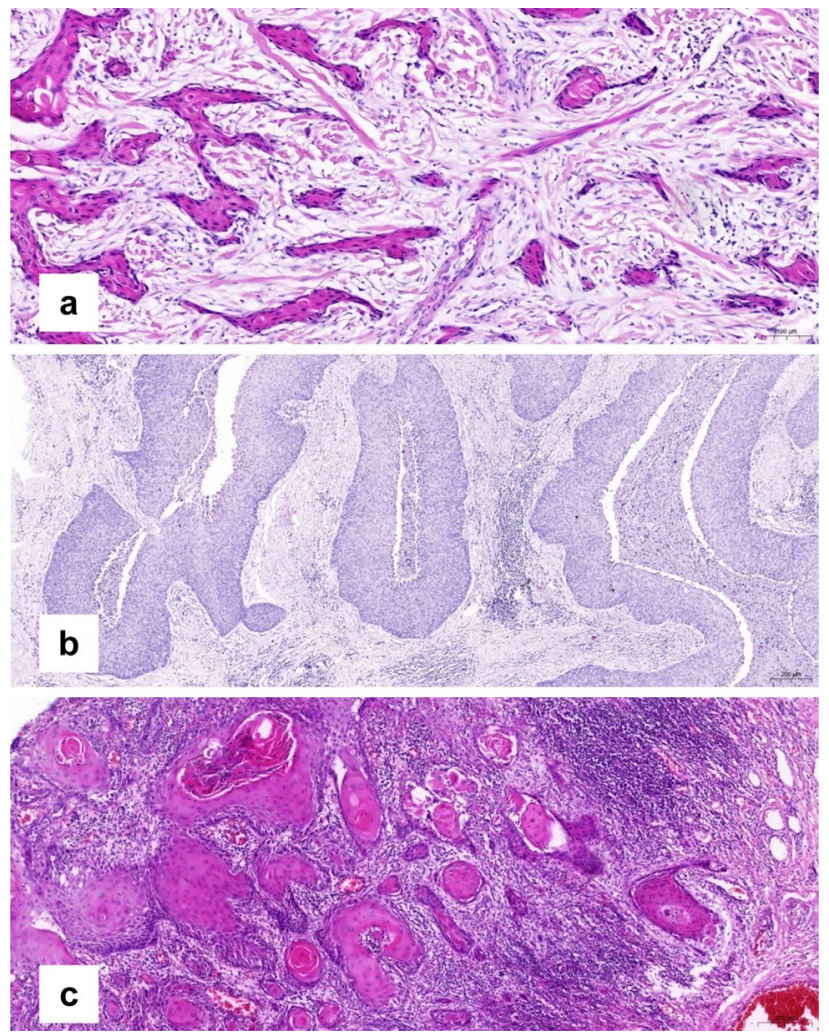

Fig. 2 Pattern of invasion and peritumoral stromal remodelling of VSCC. a Spray-like pattern of invasion and strong peritumoral desmoplastic reaction but no peritumoral inflammatory response. b Finger-like pattern of invasion without peritumoral desmoplastic reaction, very weak peritumoral inflammatory response. c Keratinizing VSCC with strong peritumoral inflammatory response

Evaluating the zonal staining within the tumor, positive staining for vimentin was restricted to the front of invasion in all cases, not the center of the tumor (Fig. 7a, b). The majority of cases $(27 / 32 ; 84.4 \%)$ showed positive staining for cyclin D1 at the front of invasion whereas the center of the tumor was negative. The opposite was observed for e-cadherin. More than two-thirds of the cases (22/32; $68.7 \%$ ) represented negative or reduced tumor cell staining at the front of invasion (Fig. 7c, d) compared to the center of the tumor.

No correlation between the tested EMT markers and the grade of peritumoral desmoplastic change, inflammatory response or the different patterns of invasion could be proven (suppl. Table 2-4). The spray-like pattern of invasion was significantly more common in $\mathrm{p} 16^{\text {non-block}} /$ p5 $3^{\text {aberrant }}$ tumors compared to $\mathrm{p} 16^{\text {block }} / \mathrm{p} 53^{\mathrm{wt}}$ tumors $(15 / 20$ (75\%) vs. $3 / 12$ (25\%); $p=0.009$; suppl. Table 1, Fig. $5 b)$.

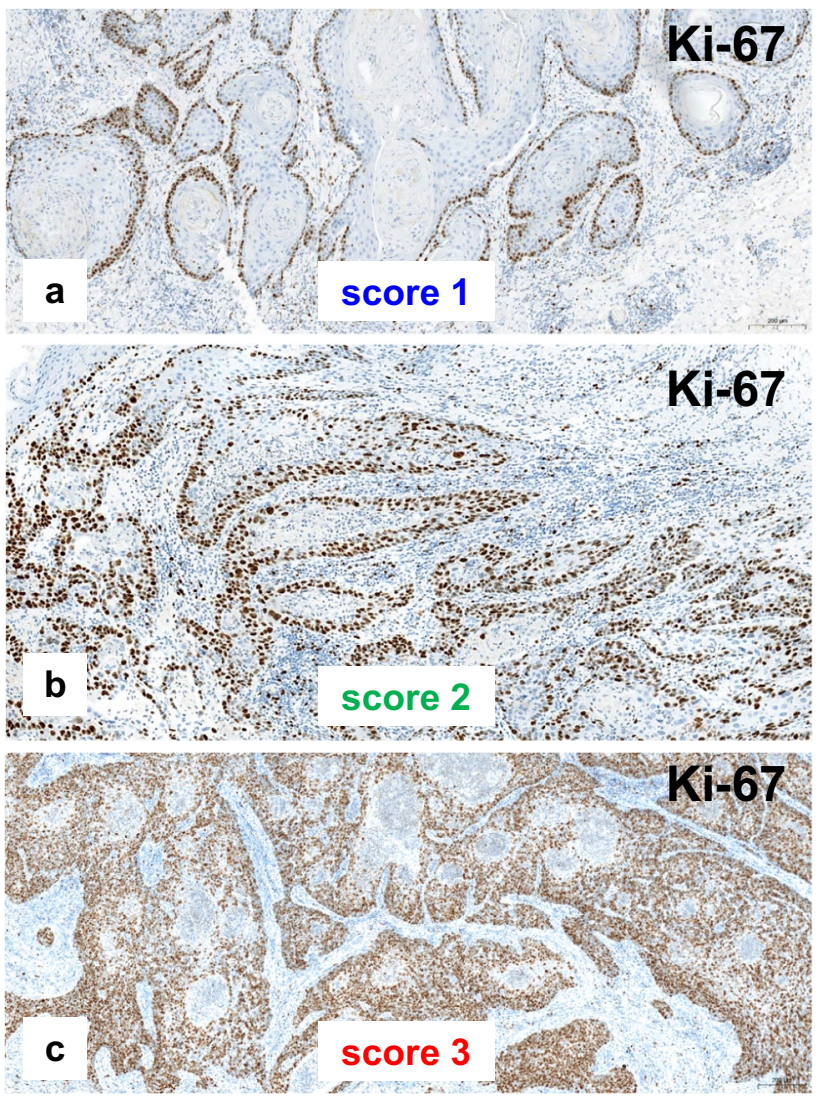

Fig. 3 Different Ki-67 scores. a, b Keratinizing VSCC with low proliferative activity. $\mathbf{c}$ Non-keratinizing VSCC with very high proliferative activity

\section{Discussion}

Lymph node involvement and recurrent disease play a critical role in the prognosis of VSCC (Rogers and Cuello 2018; Te Grootenhuis et al. 2018). Depending on the tumor stage, up to $50 \%$ of all VSCC patients will be present with lymph node involvement at the time of diagnosis (Höckel et al. 2018). Among those node positive patients, $30-50 \%$ will relapse (Melo Maia et al. 2013).

Epithelial-mesenchymal transition (EMT) has been studied in several malignancies (Bhangu et al. 2012; Toll et al. 2013), including gynecologic cancers (Stewart and McCluggage 2013). However, only few studies are available for vulvar cancer (Holthoff et al. 2016; Rodrigues et al. 2013; Zannoni et al. 2011). EMT represents a molecular shift within the tumor cells, leading to the adoption of a mesenchymal phenotype associated with loss of polarity, loss of cell adhesion and enhanced cellular motility (Toll et al. 2013), with a consecutively increased risk of metastatic spread and poor prognosis (Bhangu et al. 2012; Stewart and McCluggage 2013; Toll et al. 2013). While many immuno-histochemical markers (IHC) have been 
Table 3 Results of p53- and p16-immunostaining in correlation to proliferative activity $\left(N=31^{\mathrm{a}}\right)$

\begin{tabular}{lccc}
\hline & $\mathrm{p} 16^{\text {non-block}} / \mathrm{p} 53^{\text {aberrant }}$ & $\mathrm{p} 16^{\text {block}} / \mathrm{p} 53^{\text {wt }}$ & \\
\hline Number of cases & $20(64.5 \%)$ & $11(35.5 \%)$ & \\
Proliferative activity (Ki-67) & & & \\
Score 1 & $14(70.0 \%)$ & $2(18.2 \%)$ & \\
Score 2 & $5(25.0 \%)$ & $7(18.2 \%)$ & \\
Score 3 & $1(5.0 \%)$ & $7(63.6 \%)$ & $p<0.005$ \\
\hline
\end{tabular}

${ }^{a}$ All cases of the study could be stained immunohistochemically for vimentin and cyclin D1. In one case no tumor tissue was left within the only available tumor containing block for Ki-67 staining

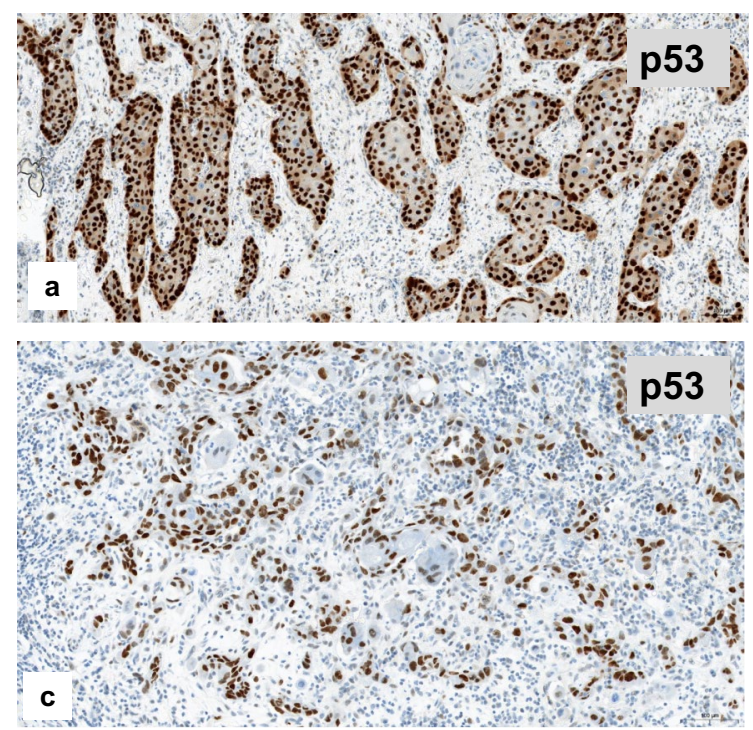

Fig. 4 p53- and p16-immunostaining as surrogate marker for the different pathogenetic pathways of VSCC. a Diffuse nuclear overexpression of p53 (i.e. aberrant expression), suggestive of a p53 mutation. b Diffuse staining for p16 (block staining), suggestive for HPV high-

evaluated to identify cells undergoing EMT, some of the most validated markers include vimentin, e-cadherin and cyclin D1 (Holthoff et al. 2016; Stewart and McCluggage 2013; Zannoni et al. 2011).

It has been shown that EMT markers are highly upregulated in VSCC tumors representing a spray-like pattern of invasion (Holthoff et al. 2016), mostly associated with a strong peritumoral desmoplastic reaction and low peritumoral inflammatory response. As reported for squamous cell carcinomas of the uterine cervix (Horn et al. 2012), a spray-like pattern of invasion is also associated with a higher risk of lymphatic spread and poor prognostic outcome in VSCC (Holthoff et al. 2016; Jeffus et al. 2015). These results could not be confirmed examining our cases (Tab. 1), may be done by the limited number of cases examined. There was no correlation of the expression of the tested EMT markers (vimentin, cyclin D-1, e-cadherin) when the different patterns of invasion were compared. The missing correlation may be biased by the limited number of cases tested.

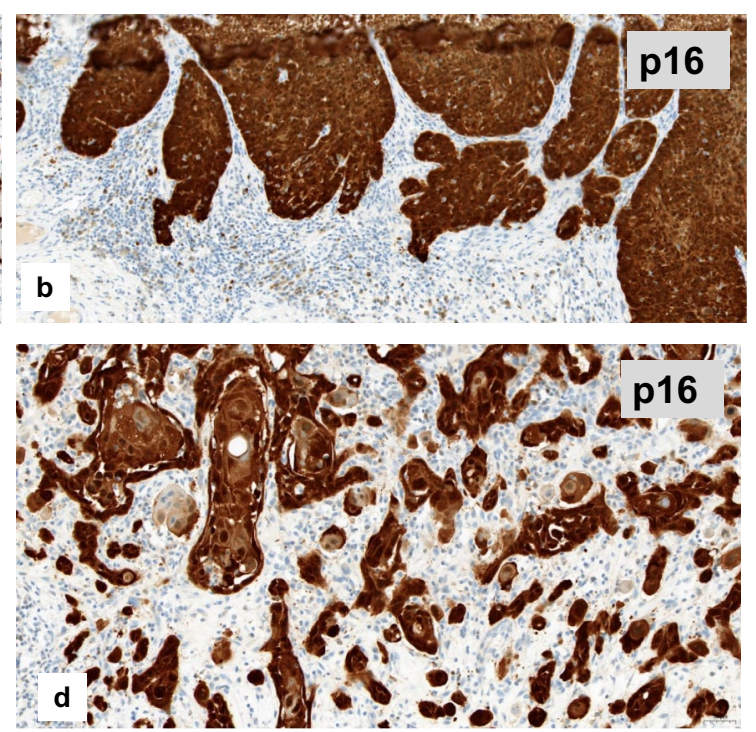

risk-associated tumor (c) and (d) so-called double classifier within a keratinizing VSCC, representing both an aberrant p53 expression and block staining for $\mathrm{p} 16$ within the same tumor

It has been shown that cells with an EMT phenotype are able to escape the immunological surveillance by host immune cells (Kudo-Saito et al. 2009). Holthoff et al. (2016) reported that VSCC with a strong peritumoral desmoplastic reaction displayed a 3.3-fold increase in the number of cases with positive staining for vimentin compared to patients with a strong peritumoral inflammatory reaction $(p=0.0031)$. In contrast, Rodrigues et al. (2013) described no correlation between the expression of e-cadherin, vimentin and other EMT-related markers and the inflammatory response in VSCC. In the present study, there was no correlation between the grade of peritumoral inflammatory response or the desmoplastic reaction and the expression of the tested EMT markers within the tumor cells (suppl. Table 2-4). In the study of Holthoff et al. (2016), there was no correlation between the loss of e-cadherin and peritumoral stromal response in VSCC. Regarding the contradictory results in a potential correlation between the peritumoral inflammatory response and the expression of EMT markers in VSCC, it 


\section{Proliferative activity} Ki-67 scoring
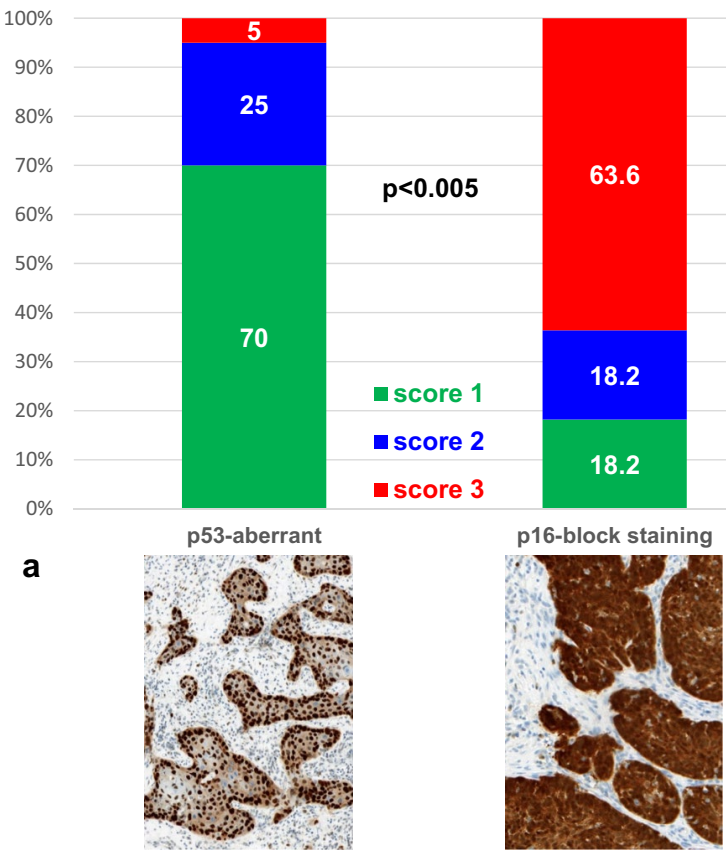

Pattern of invasion

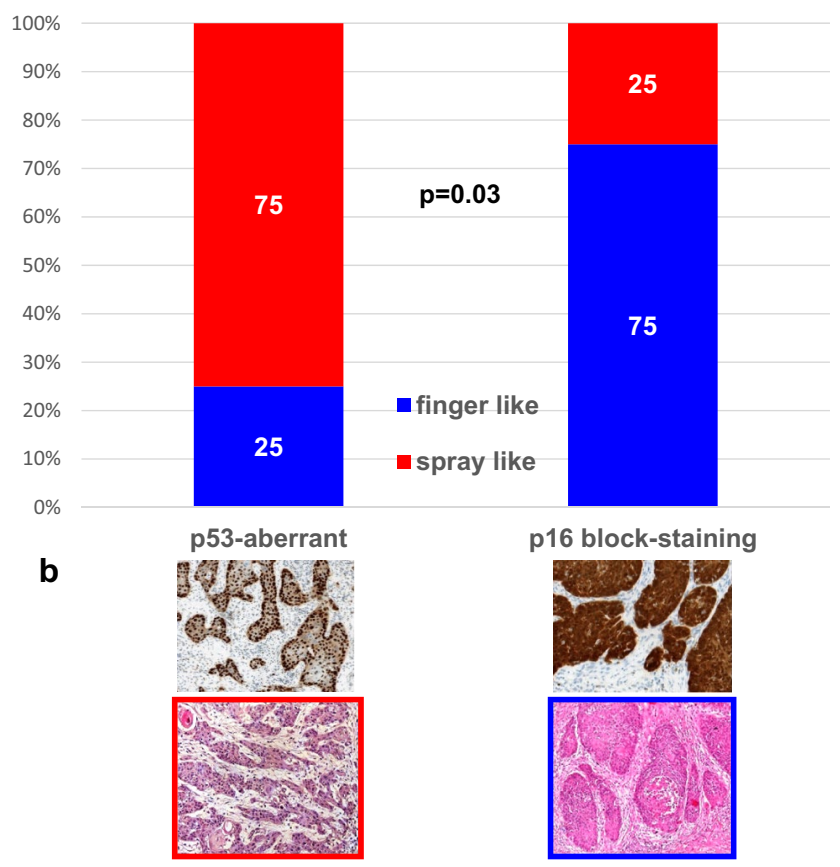

Fig. 5 Proliferative activity (Ki-67 scoring) and different patterns of invasion in correlation to immuno-histochemical p16- and p53-expression as surrogate marker for different pathogenetic pathways in VSCC (see text)
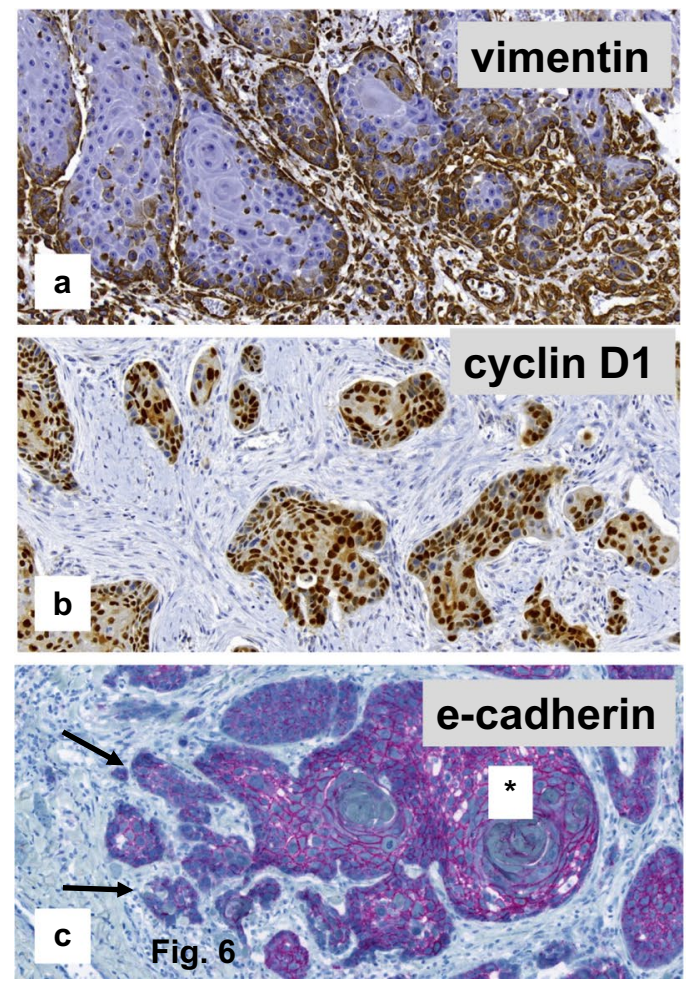

Fig.6 Immuno-expression of EMT markers within vulvar primary and inguinal lymph node metastases. a-c Primary VSCC with cytoplasmatic expression of vimentin within infiltrative tumor cell nests (a), nuclear staining for cyclin D1 (b), strong $\left(^{*}\right)$ and reduced/negative (arrow) membranous staining for e-cadherin (c), indicating epithelial-mesenchymal transition (EMT). (d-f)
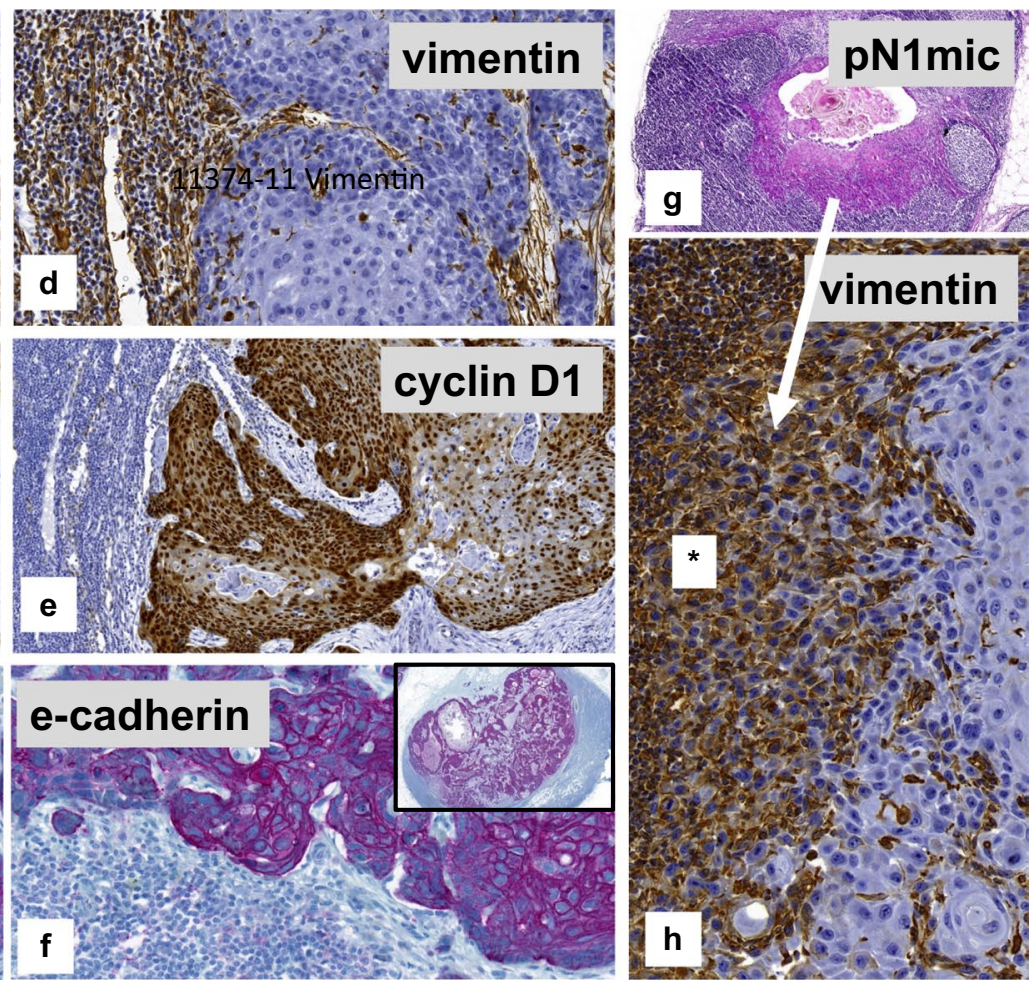

Tumor deposits within lymph node metastases representing negative staining for vimentin, nuclear positivity for cyclin D1 and diffuse staining for e-cadherin, indicating mesenchymal-epithelial transition (MET) $(\mathbf{g}, \mathbf{h})$ Micro-metastatic deposit within an inguinal lymph node (a) representing strong cytoplasmatic staining for vimentin of the majority of tumor cells $\left(^{*}\right)$ 

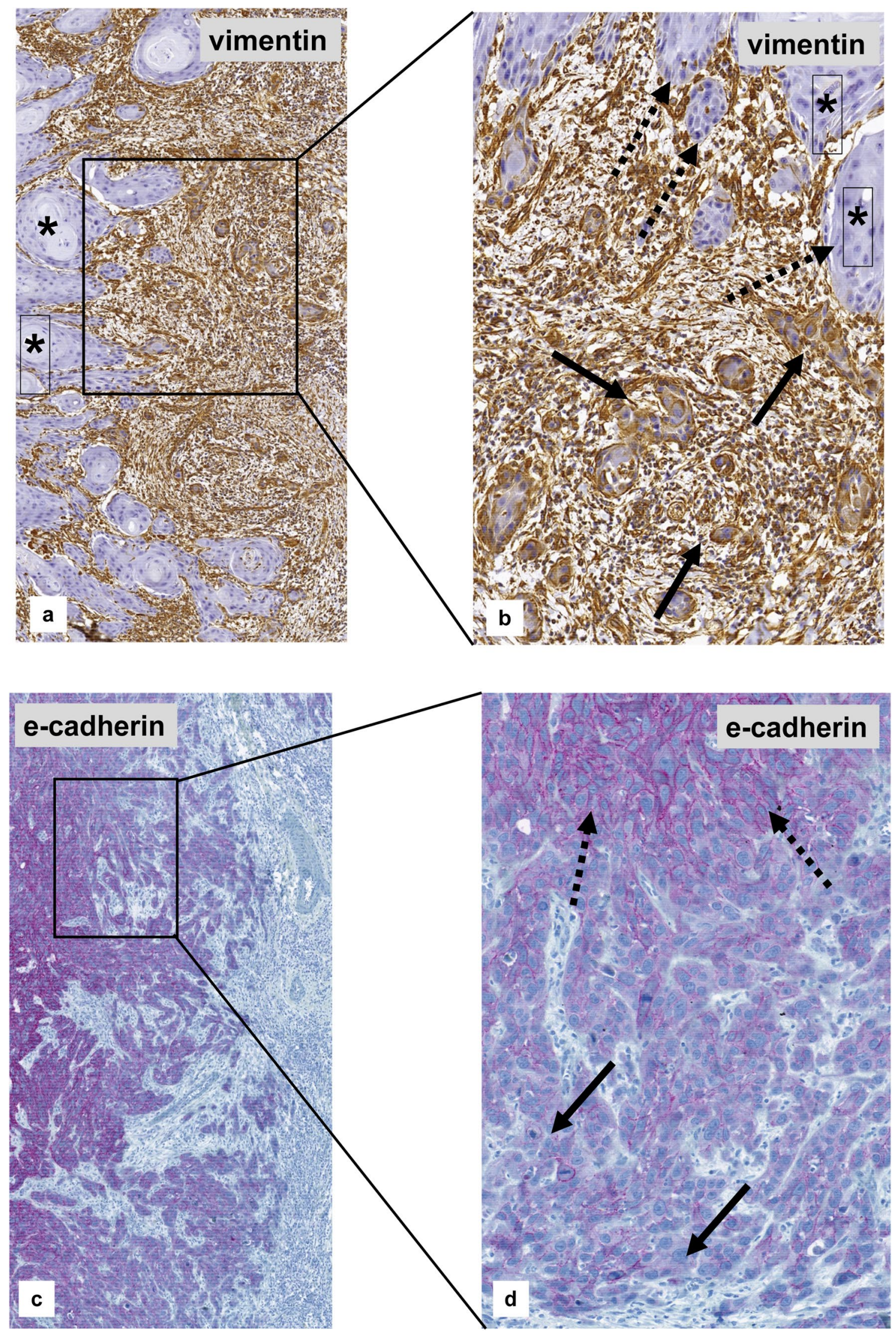
4Fig.7 Micro-anatomical EMT-immunostaining of a topographic subpopulation of tumor cells within the primary vulval VSCC. $\mathbf{a}, \mathbf{b}$ Strong cytoplasmatic staining for vimentin at the front of invasion (arrow), whereas tumor cells of the tumor center are negative $(*)$. c, $\mathbf{d}$ Strong immunostaining for e-cadherin within the center of the tumor (dotted arrow), but negative or strongly reduced membranous staining at the front of invasion (arrow)

may be hypothesized, that the presence of strong inflammatory response at the front of invasion may suppress the process of EMT in squamous cell carcinomas (Holthoff et al. 2016). Results supporting this idea are pending.

Examining HPV-negative and -positive cell lines from squamous cell carcinomas, it has been shown that HPV-I p16-positive cell lines represented a reduced EMT transition (Umbreit et al. 2014).

Rodrigues et al. (2013) described no correlation between e-cadherin and vimentin and a potential HPV infection in VSCC. However, a low $\beta$-catenin and high Slug expression were significantly associated with HPV-negative (p53-aberrant) tumors and a poor prognostic outcome. Although not reaching statistical significance, the median IRS value of vimentin was higher in VSCC with aberrant p53 staining when compared to wild type staining ( 4.5 vs. $0 ; p=0.087$; suppl. Table 1). For cyclin D1, a significantly increased staining was seen in p53-aberrant VSCC $(p=0.03)$. These results may indicate that different EMT markers may be expressed differently in VSCC depending on its pathogenetic pathways (HPV high-risk infection versus p53 mutation). In the present study, $\mathrm{p} 16^{\text {non-block}} / \mathrm{p} 53^{\text {aberrant }}$ tumors, were associated with a spray-like pattern of invasion and low proliferative activity (Ki-67 labeling score 1; Fig. 5a), likely caused by the p53 mutation. In contrast, in $\mathrm{p} 16^{\text {block }} / \mathrm{p} 53^{\mathrm{wt}} \mathrm{VSCC}$, indicating a high-risk HPV infection, finger-like pattern of invasion and high proliferative activity (score $3 \mathrm{Ki}-67$ labeling index) were significantly more common (Fig. 5b).

In squamous cell cancer of the head and neck as well as in those of the female genital tract, it has been suggested that upregulated EMT may be associated with a higher tumor stage and the presence of metastatic spread (Stewart and McCluggage 2013; Wan et al. 2020). Holthoff et al. (2016) were unable to demonstrate any correlation between the immuno-histochemical expression of the EMT markers $\beta$-catenin, vimentin and e-cadherin and nodal involvement in VSCC. Low e-cadherin expression was associated with deeper invasion, higher tumor stage and $\geq 2$ positive lymph nodes in VSCC (Rodrigues et al. 2013). In the same study, there was a significant association between positive vimentin staining and the depth of invasion, but as mentioned above, not with lymph node involvement. In the present study, there were no differences in the immuno-reactive scores of vimentin and cyclin D1 or the staining of e-cadherin between node negative and node positive cases. In the authors' opinion, additional studies are required examining a larger number of cases to compare node negative and positive VSCC. Furthermore, the cases need to be separated based on the different pathogenetic pathways of VSCC (high-risk HPV- and p53-driven tumors).

In the present study, different microanatomical locations, i.e. topographic subpopulations of tumor cells were evaluated regarding the immuno-histochemical expression of EMT markers comparing the central tumor to its front of invasion. The vast majority of cases demonstrated pronounced cyclin D1 staining at the front of invasion while vimentin staining was seen exclusively in tumor cells at the invasion front (Fig. 7a, b). Contrary, more than two-thirds of the cases represented reduced or negative staining for e-cadherin at the front of invasion (Fig. 7c, d). Holthoff et al. (2016) reported that immuno-histochemical expression of vimentin was exclusively seen at the front of invasion in two-thirds of VSCC, and 77\% displayed a loss of e-cadherin. In head and neck squamous cell carcinomas, Dal Vecchio et al. (2011) reported a significant correlation between the expression of vimentin and lymph node involvement. Rodrigues et al. (2013) found an increased vimentin expression at the front of invasion for vulvar carcinomas. In the same study, a significant loss of nuclear $\beta$-catenin staining $(p=0.013)$ was seen in tumor cells at the front of invasion, especially in HPV-negative VSCC. The infiltrative tumor cells in CIN 3-like squamous cell carcinomas of the uterine cervix showed a nuclear expression of cyclin D1 and loss of e-cadherin (Stewart and Crook 2017) whereas the central parts of the tumor demonstrated a reverse staining pattern. Both nuclear expression of cyclin D1 and loss of e-cadherin were also pictured at the front of invasion within the review by Stewart and MaCluggage (2013). Zannoni et al. (2011) reported a down-regulation of e-cadherin expression in VSCC compared to normal squamous epithelium at the front of invasion. Loss of e-cadherin leads to reduced cell adhesion which may result in an increased metastatic potential of tumor cells (Bakir et al. 2020; Jolly et al. 2017). The different zonal staining patterns suggest that the tumor cells at the front of invasion acquire an invasive and migratory behavior to support the infiltrative tumor growth and support the ability for metastatic spread. Different microanatomical staining patterns for EMT-associated markers have been reported for several gynecologic malignancies (Stewart and McCluggage 2013) including cervical squamous cell carcinomas (Koay et al. 2012; Lee and Shen 2012), suggesting that the EMT phenotype of cancer cells is a prerequisite for infiltrative tumor growth at the front of invasion as well as metastatic spread (Bakir et al. 2020; Jolly et al. 2017).

Meanwhile, EMT is believed to be a reversible process, affected by tumor microenvironment, and is followed by an inverse process, resulting in a mesenchymal-epithelial transition (MET; Bakir et al. 2020; Jolly et al. 2017; Stewart and McCluggage 2013). One hypothesis is that MET 
may be necessary for colonisation and tumor growth at the metastatic site (Guarino et al. 2007; Stewart and McCluggage 2013) as well as the realisation of metastatic deposits from circulating tumor cells (Bakir et al. 2020; Jolly et al. 2017). These ideas may be supported by the present study, showing an immuno-histochemical expression of e-cadherin in the tumor cells with on average $74.9 \%$ positively stained cells in inguinal lymph metastases of VSCC, accompanied by tumor cells that are negative for vimentin in $12 / 16$ cases within the lymph node deposits. Additionally, one of the cases with a small tumor cell deposit within the lymph node was positive for vimentin (Fig. 6h), whereas the larger metastases were completely negative. However, the number of cases included in the present study was too small to draw definitive conclusions.

The present study showed no differences in the expression of EMT markers when node positive and negative VSCC were compared. However, different staining patterns were noted within different cellular subpopulations in their micro-anatomical context indicating an EMT-phenotype in the tumor cells at the front of invasion. Paired analyses of tumor cells within the vulvar tumor and its metastatic deposits within the inguinal nodes may indicate a mesenchymal-epithelial transition (MET). Although a limited number of cases was examined, our results and those obtained from the literature suggest that EMT may be more frequent in tumors associated with a 553 mutation compared to those caused by a high-risk HPV infection. The pronounced EMT features in VSCC representing p53 alterations may be responsible for the more aggressive clinical behaviour of that tumors, regardless of lower proliferative activity.

Additional studies are required examining a larger cohort of cases with separation VSCC according to its different pathogenetic pathways. A better understanding of the etiology for lymphatic spread and metastases in HPV- and non-HPV-associated vulvar squamous cell carcinoma may eventually help identify patients at high risk for lymphatic or metastatic spread.

Supplementary Information The online version contains supplementary material available at https://doi.org/10.1007/s00432-021-03715-2.

Funding Open Access funding enabled and organized by Projekt DEAL. The authors declare that there was no funding of the study.

\section{Declarations}

Conflict of interest The authors declare that they have no conflict of interest.

Ethical approval All procedures performed in studies involving human participants were in accordance with the ethical standards of the institutional and/or national research committee and with the 1964 Helsinki declaration and its later amendments or comparable ethical standards.
Informed consent Informed consent was obtained from all individual participants included in the study.

Open Access This article is licensed under a Creative Commons Attribution 4.0 International License, which permits use, sharing, adaptation, distribution and reproduction in any medium or format, as long as you give appropriate credit to the original author(s) and the source, provide a link to the Creative Commons licence, and indicate if changes were made. The images or other third party material in this article are included in the article's Creative Commons licence, unless indicated otherwise in a credit line to the material. If material is not included in the article's Creative Commons licence and your intended use is not permitted by statutory regulation or exceeds the permitted use, you will need to obtain permission directly from the copyright holder. To view a copy of this licence, visit http://creativecommons.org/licenses/by/4.0/.

\section{References}

Bakir B, Chiarella AM, Pitarresi JR, Rustgi AK (2020) EMT, MET, plasticity, and tumor metastasis. Trends Cell Biol 30:764-776. https://doi.org/10.1016/j.tcb.2020.07.003

Bhangu A, Wood G, Mirnezami A, Darzi A, Tekkis P, Goldin R (2012) Epithelial mesenchymal transition in colorectal cancer: seminal role in promoting disease progression and resistance to neoadjuvant therapy. Surg Oncol 21:316-323. https://doi.org/10.1016/j. suronc.2012.08.003

Campo L, Zhang C, Breuer E-K (2015) EMT-inducing molecular factors in gynecological cancers. Biomed Res Int 2015:420891. https://doi.org/10.1155/2015/420891

Dal Vechio AMdC, Giudice FS, Sperandio FF, Mantesso A, Pinto Junior DdS (2011) Vimentin expression and the influence of Matrigel in cell lines of head and neck squamous cell carcinoma. Braz Oral Res 25:235-240. https://doi.org/10.1590/s1806-8324201100 0300008

Darragh TM, Colgan TJ, Thomas Cox J, Heller DS, Henry MR, Luff RD, McCalmont T, Nayar R, Palefsky JM, Stoler MH, Wilkinson EJ, Zaino RJ, Wilbur DC (2013) The Lower Anogenital Squamous Terminology Standardization project for HPV-associated lesions: background and consensus recommendations from the College of American Pathologists and the American Society for Colposcopy and Cervical Pathology. Int J Gynecol Pathol 32:76-115. https:// doi.org/10.1097/PGP.0b013e31826916c7

Dasgupta S, Ewing-Graham PC, Swagemakers SMA, van der Spek PJ, van Doorn HC, Noordhoek Hegt V, Koljenović S, van Kemenade FJ (2020) Precursor lesions of vulvar squamous cell carcinoma -histology and biomarkers: a systematic review. Crit Rev Oncol Hematol 147:102866. https://doi.org/10.1016/j.critrevonc.2020. 102866

de Melo Maia B, Munhoz Cestari F, Lavorato-Rocha AM, Sant'Ana Rodrigues I, Baiocchi G, Cardoso Guimarães G, Vassallo J, Stiepcich M, Soares FA, Malagoli Rocha R (2013) Characterization of sociodemographic and clinicopathological features in Brazilian patients with vulvar squamous cell carcinoma. Gynecol Obstet Invest 75:53-60. https://doi.org/10.1159/000343758

Guarino M, Rubino B, Ballabio G (2007) The role of epithelial-mesenchymal transition in cancer pathology. Pathology 39:305-318. https://doi.org/10.1080/00313020701329914

Höckel M, Trott S, Dornhöfer N, Horn L-C, Hentschel B, Wolf B (2018) Vulvar field resection based on ontogenetic cancer field theory for surgical treatment of vulvar carcinoma: a single-centre, single-group, prospective trial. Lancet Oncol 19:537-548. https:// doi.org/10.1016/S1470-2045(18)30109-8 
Holthoff ER, Spencer H, Kelly T, Post SR, Quick CM (2016) Pathologic features of aggressive vulvar carcinoma are associated with epithelial-mesenchymal transition. Hum Pathol 56:22-30. https:// doi.org/10.1016/j.humpath.2016.05.020

Horn L-C, Hommel N, Roschlau U, Bilek K, Hentschel B, Einenkel J (2012) Peritumoral stromal remodeling, pattern of invasion and expression of c-met/HGF in advanced squamous cell carcinoma of the cervix uteri, FIGO stages III and IV. Eur J Obstet Gynecol Reprod Biol 163:76-80. https://doi.org/10.1016/j.ejogrb.2012. 03.019

Jeffus SK, Gehlot A, Holthoff E, Stone R, Spencer H, Kelly T, Post SR, Quick CM (2015) A fibromyxoid stromal response is associated with an infiltrative tumor morphology, perineural invasion, and lymph node metastasis in squamous cell carcinoma of the vulva. Am J Surg Pathol 39:1226-1233. https://doi.org/10.1097/PAS. 0000000000000486

Jolly MK, Ware KE, Gilja S, Somarelli JA, Levine H (2017) EMT and MET: necessary or permissive for metastasis? Mol Oncol 11:755-769. https://doi.org/10.1002/1878-0261.12083

Julia CJ, Hoang LN (2021) A review of prognostic factors in squamous cell carcinoma of the vulva: evidence from the last decade. Semin Diagn Pathol 38:37-49. https://doi.org/10.1053/j.semdp. 2020.09.004

Koay MHE, Crook M, Stewart CJR (2012) Cyclin D1, E-cadherin and beta-catenin expression in FIGO Stage IA cervical squamous carcinoma: diagnostic value and evidence for epithelial-mesenchymal transition. Histopathology 61:1125-1133. https://doi.org/10. 1111/j.1365-2559.2012.04326.x

Kudo-Saito C, Shirako H, Takeuchi T, Kawakami Y (2009) Cancer metastasis is accelerated through immunosuppression during Snail-induced EMT of cancer cells. Cancer Cell 15:195-206. https://doi.org/10.1016/j.ccr.2009.01.023

Lee M-Y, Shen M-R (2012) Epithelial-mesenchymal transition in cervical carcinoma. Am J Transl Res 4:1-13

Podoll MB, Singh N, Gilks CB, Moghadamfalahi M, Sanders MA (2017) Assessment of CK17 as a marker for the diagnosis of differentiated Vulvar intraepithelial Neoplasia. Int J Gynecol Pathol 36:273-280. https://doi.org/10.1097/PGP.0000000000000317

Protzel C, Knoedel J, Zimmermann U, Woenckhaus C, Poetsch M, Giebel J (2007) Expression of proliferation marker Ki67 correlates to occurrence of metastasis and prognosis, histological subtypes and HPV DNA detection in penile carcinomas. Histol Histopathol 22:1197-1204

Rodrigues IS, Lavorato-Rocha AM, Maia M, B de, Stiepcich MMA, Carvalho FM de, Baiocchi G, Soares FA, Rocha RM, (2013) Epithelial-mesenchymal transition-like events in vulvar cancer and its relation with HPV. Br J Cancer 109:184-194. https://doi.org/ $10.1038 / \mathrm{bjc} .2013 .273$

Rogers LJ, Cuello MA (2018) Cancer of the vulva. Int J Gynaecol Obstet 143(Suppl 2):4-13. https://doi.org/10.1002/ijgo.12609

Singh N, Gilks CB (2020) Vulval squamous cell carcinoma and its precursors. Histopathology 76:128-138. https://doi.org/10.1111/ his. 13989

Stankiewicz E, Ng M, Cuzick J, Mesher D, Watkin N, Lam W, Corbishley C, Berney DM (2012) The prognostic value of Ki-67 expression in penile squamous cell carcinoma. J Clin Pathol 65:534-537. https://doi.org/10.1136/jclinpath-2011-200638

Stewart CJR, Crook ML (2017) Cervical intraepithelial neoplasia (CIN) 3-like squamous cell carcinoma of the cervix: a review of 14 cases with comparison of E-cadherin and cyclin D1 expression in the CIN 3-like and infiltrative tumour elements. Histopathology 70:367-374. https://doi.org/10.1111/his.13094

Stewart CJR, McCluggage WG (2013) Epithelial-mesenchymal transition in carcinomas of the female genital tract. Histopathology 62:31-43. https://doi.org/10.1111/his. 12057

Te Grootenhuis NC, Pouwer A-FW, de Bock GH, Hollema H, Bulten J, van der Zee AGJ, de Hullu JA, Oonk MHM (2018) Prognostic factors for local recurrence of squamous cell carcinoma of the vulva: a systematic review. Gynecol Oncol 148:622-631. https:// doi.org/10.1016/j.ygyno.2017.11.006

Tessier-Cloutier B, Kortekaas KE, Thompson E, Pors J, Chen J, Ho J, Prentice LM, McConechy MK, Chow C, Proctor L, McAlpine JN, Huntsman DG, Gilks CB, Bosse T, Hoang LN (2020) Major p53 immunohistochemical patterns in in situ and invasive squamous cell carcinomas of the vulva and correlation with TP53 mutation status. Mod Pathol 33:1595-1605. https://doi.org/10.1038/ s41379-020-0524-1

Toll A, Masferrer E, Hernández-Ruiz ME, Ferrandiz-Pulido C, Yébenes M, Jaka A, Tuneu A, Jucglà A, Gimeno J, Baró T, Casado B, Gandarillas A, Costa I, Mojal S, Peña R, de Herreros AG, García-Patos V, Pujol RM, Hernández-Muñoz I (2013) Epithelial to mesenchymal transition markers are associated with an increased metastatic risk in primary cutaneous squamous cell carcinomas but are attenuated in lymph node metastases. J Dermatol Sci 72:93-102. https://doi.org/10.1016/j.jdermsci.2013.07.001

Umbreit C, Flanjak J, Weiss C, Erben P, Aderhold C, Faber A, SternStraeter J, Hoermann K, Schultz JD (2014) Incomplete epithelial-mesenchymal transition in p16-positive squamous cell carcinoma cells correlates with $\beta$-catenin expression. Anticancer Res 34:7061-7069

Wan Y, Liu H, Zhang M, Huang Z, Zhou H, Zhu Y, Tao Y, Xie N, Liu X, Hou J, Wang C (2020) Prognostic value of epithelialmesenchymal transition-inducing transcription factors in head and neck squamous cell carcinoma: a meta-analysis. Head Neck 42:1067-1076. https://doi.org/10.1002/hed.26104

Zannoni GF, Prisco MG, Vellone VG, de Stefano I, Scambia G, Gallo D (2011) Changes in the expression of oestrogen receptors and E-cadherin as molecular markers of progression from normal epithelium to invasive cancer in elderly patients with vulvar squamous cell carcinoma. Histopathology 58:265-275. https://doi.org/ 10.1111/j.1365-2559.2011.03744.x

Publisher's Note Springer Nature remains neutral with regard to jurisdictional claims in published maps and institutional affiliations. 